\title{
Usefulness of percutaneous microwave ablation for large non-small cell lung cancer: A preliminary report
}

\author{
CLAUDIO PUSCEDDU ${ }^{1}$, LUCA MELIS ${ }^{1}$, BARBARA SOTGIA ${ }^{1}$, DANIELA GUERZONI ${ }^{2}$, \\ ALBERTO PORCU $^{3}$ and ALESSANDRO FANCELLU ${ }^{3}$
}

\begin{abstract}
${ }^{1}$ Division of Interventional Radiology, Department of Oncological Radiology; ${ }^{2}$ Department of Medical Oncology, Oncological Hospital A. Businco, I-09121 Cagliari; ${ }^{3}$ Department of Clinical, Surgical and Experimental Sciences, Unit of General Surgery 2-Clinica Chirurgica, University of Sassari, I-07100 Sassari, Italy
\end{abstract}

Received January 20, 2019; Accepted May 13, 2019

DOI: $10.3892 / 01.2019 .10375$

\begin{abstract}
The role of microwave ablation (MWA) in patients with non-small cell lung cancer (NSCLC) remains ill-defined. This retrospective study evaluated the oncological outcomes of CT-guided MWA in patients with large NSCLC. Kaplan-Meier analysis was used to evaluate overall survival (OS) and cancer-specific survival (CSS). The log-rank test was used to compare survival between patients with an NSCLC size greater or smaller than $4 \mathrm{~cm}$. The likelihood of local tumor progression (LTP) was analyzed using a multivariable regression model. A total of 53 patients with 65 tumors were analyzed. The mean tumor size was $5.0 \pm 1.8 \mathrm{~cm}$. At the 1 -month CT scan, complete tumor ablation was observed in $44.6 \%$ of cases. In $18.5 \%$ of cases a redo-MWA session was carried out, while in $4.6 \%$, a third MWA was necessary to obtain complete tumor necrosis. The mean follow-up was $28.1 \pm 20.6$ months with a median duration of 21.5 months. The 1-year, 2-year, 3-year and 5-year OS rates were $78.2,48.3,34.8$ and $18.3 \%$, respectively. The median CSS was 25 months (95\% CI 15.5-34.5). The 1-year, 2-year, 3-year and 5-year CSS rates were 84.3, 53.7, 42.1 and $30.0 \%$, respectively. OS in patients with tumor size $\geq 4 \mathrm{~cm}$ was significantly lower when compared with those having smaller tumors $(\mathrm{P}=0.03)$. LTP was observed in 19 patients $(35.8 \%)$. Incomplete tumor ablation [odds ratio (OR) 6.57; $\mathrm{P}<0.05$ ] and tumor size $\geq 4 \mathrm{~cm}(\mathrm{OR} 0.18 ; \mathrm{P}<0.05)$ were significant independent predictors of LTP. In conclusion, CT-guided MWA may represent a useful tool in the multimodality treatment of patients with large advanced NSCLC.
\end{abstract}

Correspondence to: Professor Alessandro Fancellu, Department of Clinical, Surgical and Experimental Sciences, Unit of General Surgery 2-Clinica Chirurgica, University of Sassari, V.le San Pietro 43, I-07100 Sassari, Italy

E-mail: afancel@uniss.it

Key words: non-small cell lung cancer, microwave ablation, CT-guided ablation, non-surgical treatment, percutaneous treatment, multimodality cancer treatment

\section{Introduction}

Lung cancer remains the leading cause of cancer-related mortality in the world. More than 180,000 men and 90,000 women succumb to lung cancer every year within the European Union (1). Non-small-cell lung cancer (NSCLC) accounts for $>80 \%$ of all lung cancer cases (2). Surgical resection represents the treatment of choice for patients with NSCLC at stages I and II, and can also be used for some patients with locally advanced disease (stage IIIA and IIIB) as an important component of the multimodal treatment approach. In general, only $20-25 \%$ of patients with NSCLC are suitable for surgical resection at the time of diagnosis $(3,4)$. For those with advanced disease, radiation therapy, chemotherapy and targeted therapies are commonly used $(2,5)$. However, despite the fact that the multimodal treatment approach has improved outcomes over the last 20 years, the 5-year survival rate for stage III patients still ranges between 13 and 36\% (6).

In the last ten years, there has been a surge of ablative treatments for solid tumors, which have been shown to be effective in patients not suitable for surgery (7-14). In particular, thermal ablation techniques, such as radiofrequency ablation, microwave ablation (MWA), cryoablation and laser ablation, have been used with the aim of treating primary and secondary lung tumors in a minimally invasive manner (9,15-20). Among them, MWA can be considered a relatively new method, in which one or more microwave antennas are inserted percutaneously inside the tumor mass. The electromagnetic microwaves produce thermal energy that causes coagulative necrosis of neoplastic cells and the surrounding parenchyma $(10,14,18,21)$. MWA offers some advantages when compared to radiofrequency ablation, which has been the most used method for ablation of nonresectable lung NSCLC $(8,15,22)$. In fact, radiofrequency is associated with some limitations and disadvantages, such as reduced efficacy due to increased impedance from a temperature $>100^{\circ} \mathrm{C}$, and the heat sink effect. By contrast, the efficacy of MWA ablation is not affected by high temperatures and seems less affected by the heat sink effect $(8,10,11,15)$. Moreover, two or more antennae can be used when necessary to obtain a larger ablation zone in a shorter time $(4,8,10,11,22,23)$. A multicenter study, including 52 patients with lung cancer, comparing 
radiofrequency ablation and MWA reported that the latter was associated with less intraprocedural pain and a significant reduction in tumor mass (24).

Although evidence has emerged that MWA is a promising treatment option for primary and metastatic lung malignancies, long-term follow-up data are scant. This study was performed with the main aim of evaluating the survival outcomes of patients with large advanced NSCLC undergoing MWA under CT guidance.

\section{Patients and methods}

Study population. The present study was conducted at an oncological institution with expertise in interventional radiology, where different ablative techniques have been used in the last decade for thermal ablation of either primary or metastatic malignancies of the liver, pancreas, bone, breast and lung. For the purposes of this study, a retrospective review was conducted on NSCLC patients receiving MWA between 2010 and 2013. The Institutional Review Board of the Division of Interventional Radiology, Department of Oncological Radiology, Oncological Hospital A. Businco, Cagliari (Italy) approved the present study. All patients gave informed written consent for the MWA treatment.

Indications for lung MWA included one or more of the following: i) NSCLC at stage IIIb-IIIc; ii) NSCLC not suitable for surgical treatment because of poor general status or concomitant medical conditions; iii) advanced NSCLCs not responding to chemotherapy and/or radiotherapy; iv) patients with operable disease refusing surgical treatment. Exclusion criteria included: Primary lung tumor different from the NSCLC (e.g., small-cell lung cancer and neuroendocrine tumors); tumors infiltrating large vascular structures, the trachea or esophagus; parietal pleural transgression into the chest wall; severe pulmonary dysfunction (maximum ventilation capacity $<39 \%$ ); platelet count of $<50,000$ per microliter; and/or unmanageable coagulation disorders.

The following data were extrapolated for the entire study population: Age, sex and tumor characteristics (size, number and localization of lesions, histotype and vicinity to relevant anatomical structures), as well as ablation technique details and MWA-related complications. Follow-up data included the occurrence of local tumor recurrence, survival time and cause of mortality. Patients with at least 3 months follow-up were eligible.

Preoperative work-up. Patients received a pre-procedural visit approximately 2 weeks (10-20 days) before MWA. Staging of all patients included: Histologic confirmation of NSCLC, chest radiography, contrast-enhanced total body CT-scan, lung function tests, cardiological assessment, complete blood counts and a coagulation study. Only patients with a life expectancy of $>3$ months were considered for MWA. When present, anticoagulative and antiplatelet medications were stopped 2 days and 1 week before the procedure, respectively. Risks and benefits of the MWA procedure were discussed with all patients, and informed consent was obtained from all individual participants included in the study.

MWA procedure. All MWA treatments were carried out under CT guidance (Somatom Sensation Unit; Siemens,
Germany) by board-certified interventional radiologists. The patients were maintained in a state of conscious sedation by using intravenous fentanyl citrate, and their vital signs (oximetry, blood pressure and heart rate) were continuously monitored throughout the procedure. After sterile preparation of the skin, 2-5 $\mathrm{ml}$ of $2 \%$ lidocaine was injected into the deep subcutaneous tissue along the expected course of the MWA antenna. Percutaneous MWA was performed with patients in the prone or supine position by using a $2.45-\mathrm{GHz}$ microwave generator (AMICA-GEN; HS Hospital Service, Aprilia, Italy) with energy delivered via 14- or 16-gauge mini-choked, water-cooled interstitial antennae (HS AMICA; HS Hospital Service). For treatment of synchronous tumor lesions, two generators were used, each driving a single antenna. The duration of each MWA application was based on the size and location of the lesion measured by the pre-operative CT scan. The antenna was located into the tumor and connected to the generator. In all cases, the positioning of the antenna within the lesion was performed carefully in order to avoid non-target thermal damage to other anatomical structures (e.g., aorta or bronchus). The number and type of antenna were chosen at the operator's discretion according to several factors, such as the 'access window', tumor size, morphology, tumor location and adjacent structures. CT-guided MWA was performed with a single antenna for tumors of $3-4 \mathrm{~cm}$ in diameter and with two antennae for tumors $>4 \mathrm{~cm}$. The time for energy deposition ranged from 5-10 mins and the ablation power varied between 40 and $80 \mathrm{~W}$. When technically possible, the MWA procedure was performed with the aim of encompassing the lung tumor with an ablation zone at least $5 \mathrm{~mm}$ of safety margin.

Post-procedural imaging and follow-up evaluation. Assessment of MWA efficacy was made by CT scan at 1 , 3, 6 and 12 months for the first year after treatment, and every 6 months thereafter. Tumor ablation was deemed to be complete when no contrast enhancement was observed in the arterial phase at the 1-month follow-up CT, whereas ablation was considered incomplete when a residual contrast enhancement was observed (Fig. 1). The size of the ablation zone measured at the 1-month CT scan was used as the basal assessment to which subsequent follow-up imaging was compared. Increases in the diameter of the MWA ablation zone were considered as tumor progression, while a plateau or decrease in the diameter of the ablation zone was interpreted as successful ablation. In some cases of residual ablation, a second MWA session was performed.

Statistical analysis. Continuous patient and tumor data are presented as the mean, range and standard deviation. The three patients with early NSCLC who underwent MWA because of surgery refusal were excluded from the survival analysis. Kaplan-Meier analysis was used to evaluate overall survival (OS) and cancer-specific survival (CSS), which were calculated from the date of treatment to the date of mortality or final follow-up. The log-rank test was used to compare survival data between patients having an NSCLC size greater or smaller than $40 \mathrm{~mm}$ in maximum diameter. The likelihood of local tumor progression (LTP) was analyzed through a multivariable logistic regression model. Tumor size was dichotomized to a binary variable $(<4 \mathrm{~cm}$ vs. $\geq 4 \mathrm{~cm})$ as well as 

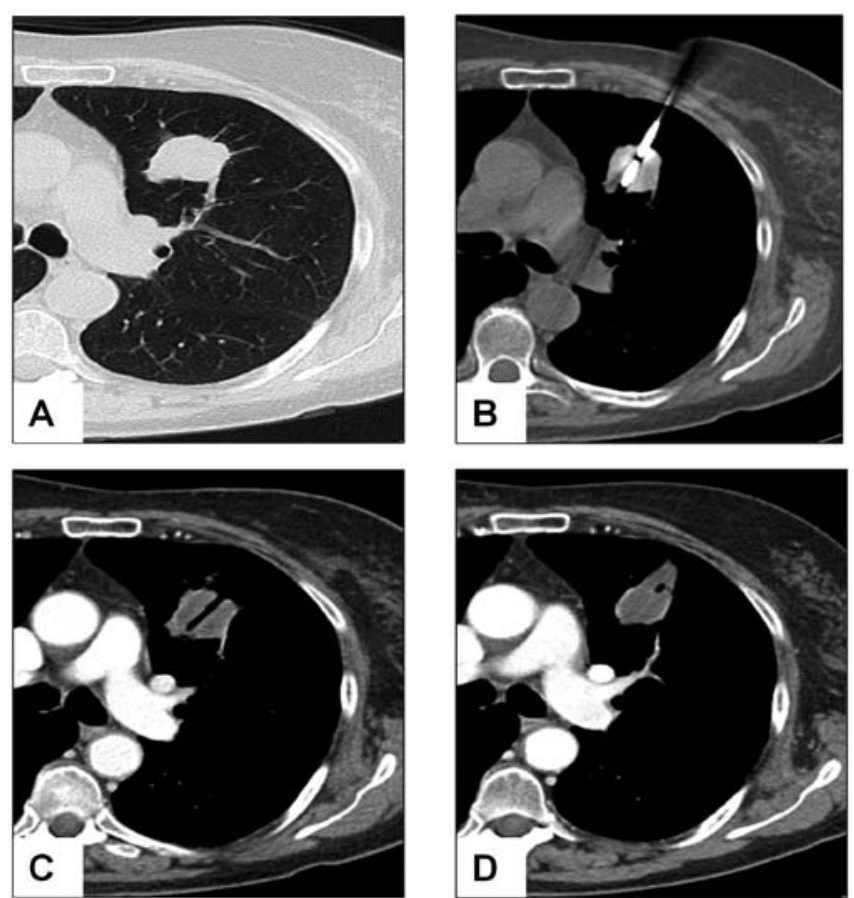

Figure 1. Complete MWA of adenocarcinoma measuring $3.7 \mathrm{~cm}$ in major diameter of the left lung in a 71-year-old woman. (A) Basal contrast-enhanced CT scan. (B) MWA by insertion of one antenna with an output power of $60 \mathrm{~W}$ for $10 \mathrm{~min}$. (C) CT control $24 \mathrm{~h}$ after the procedure. (D) Follow-up CT scan at 1 month, showing a tumor size reduction without any evidence of enhancement. MWA, microwave ablation.

the remaining covariates (proximity to relevant structures, yes vs. no; and complete vs. incomplete tumor necrosis after first MWA treatment). Covariates were chosen based on clinical significance. For each variable, a reference category was chosen, generally the no-exposure or majority category, and the other category was compared with the reference category. The odds ratios (ORs) in each category vs. the odds in the reference category were estimated. The goodness of fit of the model was assessed by the Hosmer and Lemeshow test, and $\mathrm{P}<0.05$ was considered to indicate a statistically significant difference. Analysis was conducted using IBM SPSS Statistics v.20 (IBM Corp.).

\section{Results}

Patients and tumours characteristics. From January 2010 to December 2013, 53 patients with primary NSCLC lesions consecutively underwent percutaneous MWA treatment under CT guidance. Patients and tumor characteristics are summarized in Table I. The mean age was 70.3 years (median 70.5 years), and $86 \%$ of patients were male. A total of 50 patients $(94.3 \%)$ were considered not suitable for surgery after a multidisciplinary team discussion, and 3 patients $(5.7 \%)$ with early-stage disease refused surgical treatment. Prior to MWA treatment, among the 50 patients unsuitable for surgery, $16(32.0 \%)$ patients received chemotherapy as primary treatment. Two patients (4\%) refused pharmacologic treatment, and $32(64 \%)$ were excluded from chemotherapy due to advanced age or coexisting comormibidities. MWA was used as a further treatment in patients in whom chemotherapy failed to significantly reduce the tumor size.
Table I. Patient and tumor characteristics.

$\begin{array}{ll}\text { Characteristics } & \text { No. (\%) }\end{array}$

Patients submitted to MWA for NSCLC 53

Age, mean \pm SD (range)

$70.3 \pm 10.0(43-84)$

Sex

Male

$43(86 \%)$

Female

$7(14 \%)$

MWA treatment intention

Palliative

$50(94.3)$

Curative

$16(32 \%)$

Chemotherapy as primary

treatment in patients at IIIa/IIIb stages

Total no. of NSCLC nodules treated

$65(100 \%)$

with MWA

Single NSCLC

$53(81.5 \%)$

2 nodules of NSCLC

$12(18.5 \%)$

Tumor location

Central

$16(24.6 \%)$

Peripheral

$49(75.4 \%)$

$11(16.9)$

anatomical structures

Tumor size, mean \pm SD (range)

$3-4 \mathrm{~cm}$

$5.0 \pm 1.8(3.0-11.0)$

$26(40.0 \%)$

$39(60.0 \%)$

T stage

$\mathrm{T} 2 \mathrm{a}$

$26(40.0 \%)$

$\mathrm{T} 2 \mathrm{~b}$

$14(21.5 \%)$

T3

$17(26.2 \%)$

T4

$8(12.3 \%)$

Histotype

Squamous cell carcinoma

$13(20.0 \%)$

Adenocarcinoma

$51(78.4 \%)$

$1(1.6 \%)$

Large cell carcinoma

$22(33.8 \%)$

Right upper lobe

$14(21.6 \%)$

Right lower lobe

$16(24.6 \%)$

Left upper lobe

$13(20.0 \%)$

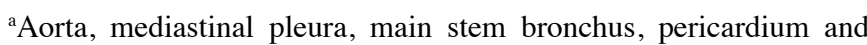
diaphragm. MWA, microwave ablation; NSCLC, non-small cell lung cancer.

MWA treatment. A total of 12 patients had 2 NSCLC lesions, thus there were a total of 65 MWA treatment sessions. Most of the tumors $(75.4 \%)$ had a peripheral lung location. At baseline, mean tumor size was $5.0 \pm 1.8 \mathrm{~cm}$ (median size $4.6 \mathrm{~cm}$ ), being the $60 \%$ of cases $>4 \mathrm{~cm}$ in major diameter (Fig. 2). As for histological type, 51 (78.4\%) of the tumors were adenocarcinomas, $13(20 \%)$ squamous cell carcinomas, and $1(1.6 \%)$ large cell carcinoma. More than one-half of the NSCLC tumors were located in the right lung. Out of 65 tumors, 11 

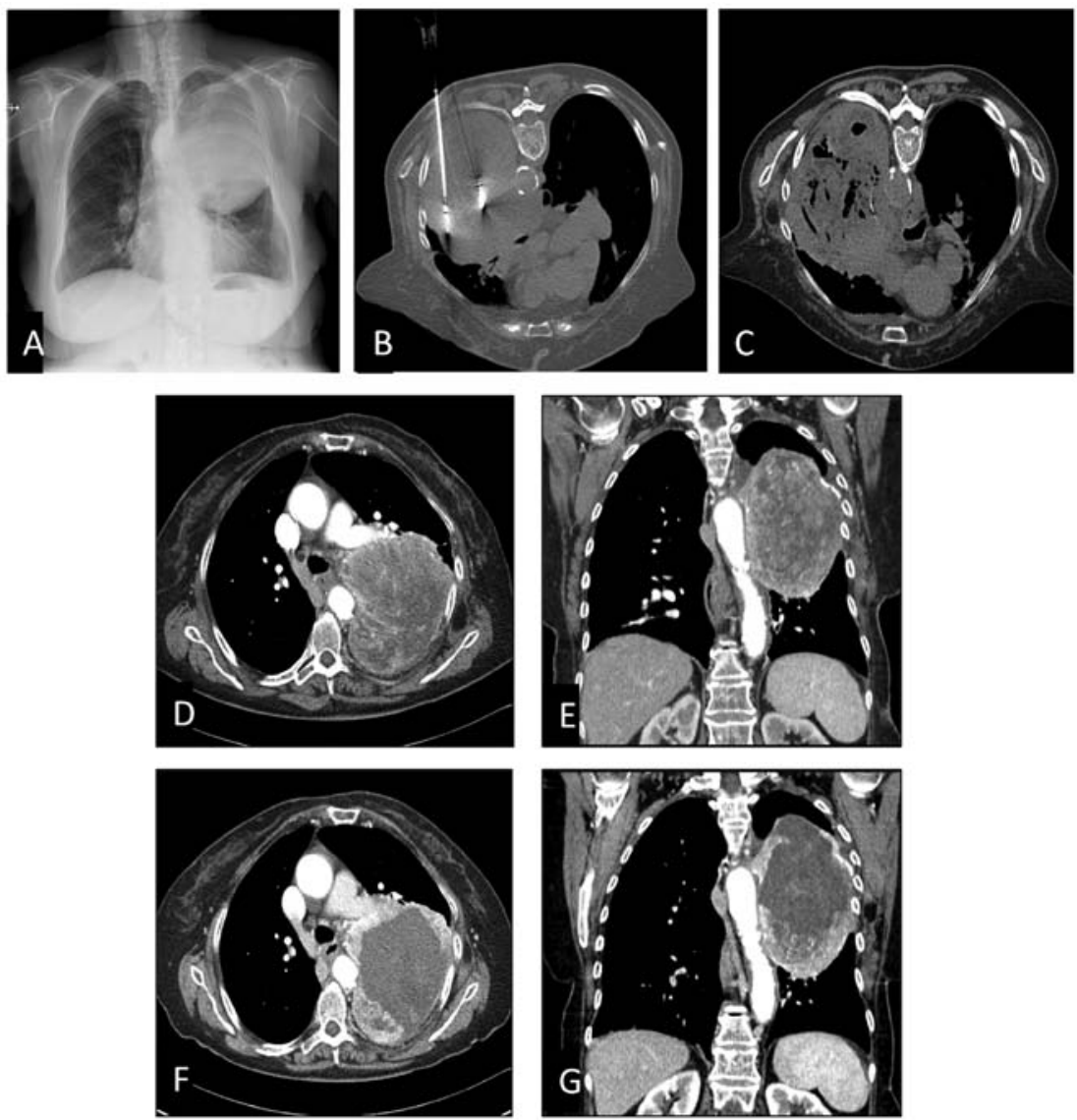

Figure 2. MWA of a large adenocarcinoma of the left lung in a 78-year old woman. (A) Chest x-ray demonstrating a $14 \mathrm{x} 10 \mathrm{~cm}$ mass in the upper lobe of the left lung. (B) CT-guided MWA using two antennae with multiple insertions. (C) CT scan after MWA treatment showing a large area of cavitation, suggestive of tumor necrosis. (D) Pre-treatment transversal and coronal (E) CT scan showing a large mass in the upper left lobe, in close proximity to large mediastinal vessels and parietal pleura. (F) Transversal and coronal (G) CT scan after 1 month showing extensive necrosis with residual contrast-enhancement. The patient succumbed 7 months after the treatment. MWA, microwave ablation.
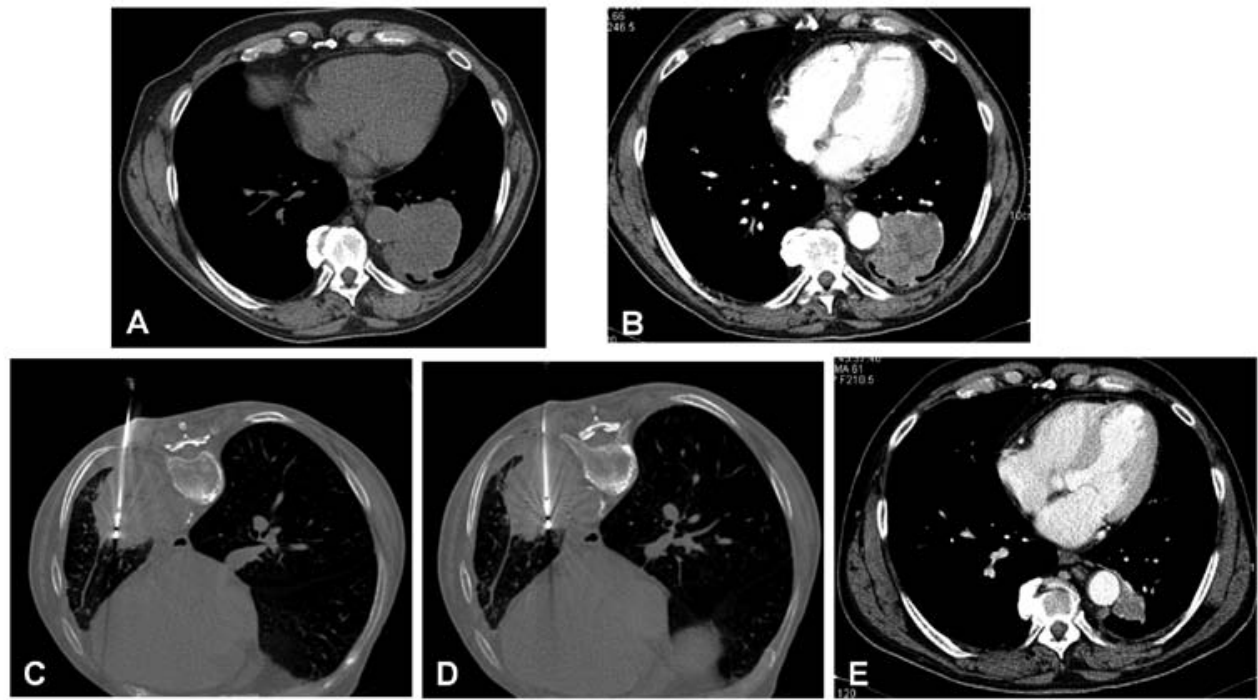

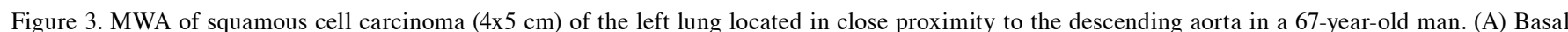
unenhanced CT scan and (B) contrast-enhanced CT scan. (C) MWA by insertion of an antenna with output power of $70 \mathrm{~W}$ for 10 min with patient in prone position. (D) MWA by insertion of a second antennae with an output power of $70 \mathrm{~W}$ for 10 min with the patient in a prone position. (E) Follow-up CT scan at 10 months showing a tumor size reduction $(1.8 \mathrm{~cm})$ with subtle enhancement. MWA, microwave ablation.

$(19.6 \%)$ were in proximity to relevant structures such as the aorta, mediastinal pleura, main stem bronchus, pericardium or diaphragm (Fig. 3).
All procedures were successfully completed. Complications occurred after $18(27.7 \%)$ procedures, all of which were resolved conservatively except in one patient, who developed 
Table II. Summary of MWA procedures and combinatorial treatment.

\begin{tabular}{lc}
\hline Characteristics & No (\%) \\
\hline No. of antennas used per single procedure & \\
1 & $39(60.0)$ \\
2 & $26(40.0)$ \\
Initial response to MWA & \\
Complete tumor ablation & $29(44.6)$ \\
Partial tumor ablation & $36(55.4)$ \\
Second session of MWA & $12(18.5)$ \\
Third session of MWA & $3(4.6)$ \\
Total procedure-related complication & $18(27.7)$ \\
Pneumothorax treated conservatively & $11(16.9)$ \\
Pneumothorax treated with tube thoracostomy & $1(1.5)$ \\
Pleural effusion & $2(3.1)$ \\
Cavitation and infection & $3(4.6)$ \\
Bronchopleural fistula & $1(1.5)$ \\
Combinatorial treatment used along with MWA & $17(32.1)$ \\
Chemotherapy & $11(20.7)$ \\
Radiation therapy & $4(7.5)$ \\
Chemotherapy + Radiation therapy & $2(83.8)$ \\
\hline
\end{tabular}

MWA, microwave ablation.

a pneumothorax needing tube thoracostomy. The overall 30 -day mortality rate was $0 \%$. At the 1 -month CT scan, a complete tumor ablation was observed after 29 MWA procedures (44.6\%), and a partial tumor ablation after 36 (55.4\%). In 12 cases $(18.5 \%)$ a redo-MWA session was carried out to obtain complete necrosis, while in 3 cases (4.6\%) a third MWA was necessary (Table II). Combinatorial treatment used along with MWA involved 17 patients (32.1\%), of whom 11 received second-line chemotherapy, 4 radiation therapy and 2 chemotherapy plus radiation therapy.

Follow-up. The mean follow-up time was $28.10 \pm 20.6$ months with a median duration of 21.5 months (range, 3-84 months). A total of 35 patients succumbed during the considered follow-up period. Median OS was 20.0 months (95\% confidence interval, 12.38-27.61). The 1-year, 2-year, 3-year and 5-year OS rates were $78.2,48.3,34.8$ and $18.3 \%$, respectively (Fig. 4). Overall CSS was 25 months (95\% confidence interval, 15.47-34.52). The 1-year, 2-year, 3-year and 5-year CSS rates were 84.3, 53.7, 42.1 and $30.0 \%$, respectively (Fig. 5). Overall survival in patients with a tumor size $\geq 4 \mathrm{~cm}$ was significantly lower when compared with patients with smaller tumors ( $\mathrm{P}=0.03$; Fig. 6$)$. LTP was observed in 19 patients $(35.8 \%)$. According to the multivariable analysis, incomplete tumor ablation [odds ratio (OR), 6.57; $\mathrm{P}<0.05$ ] and a tumor size $\geq 4 \mathrm{~cm}(\mathrm{OR}, 0.18 ; \mathrm{P}<0.05)$ were significant predictors of LTP (Table III).

\section{Discussion}

Most patients with NSCLC are commonly diagnosed at an advanced stage, and are not candidates for surgical

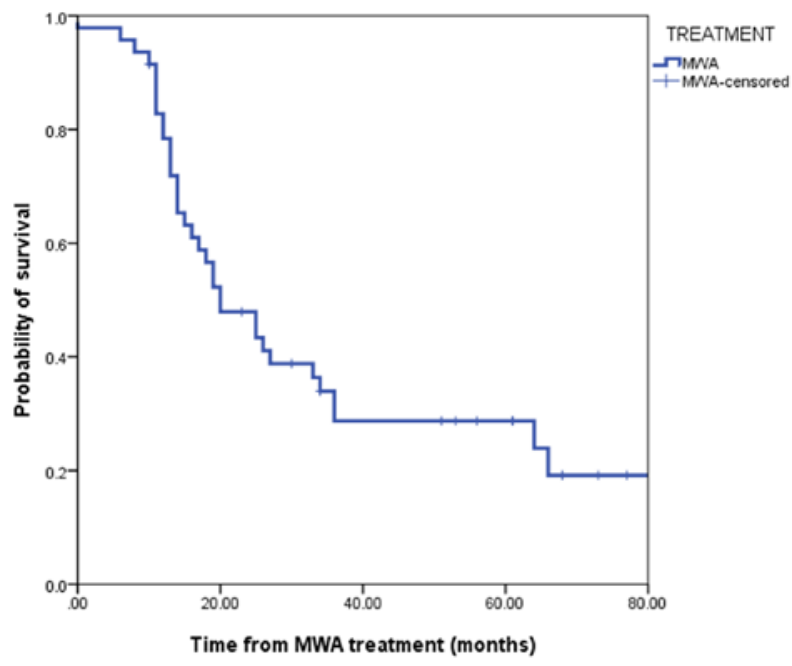

Figure 4. Overall survival after MWA for non-small-cell lung cancer. MWA, microwave ablation.

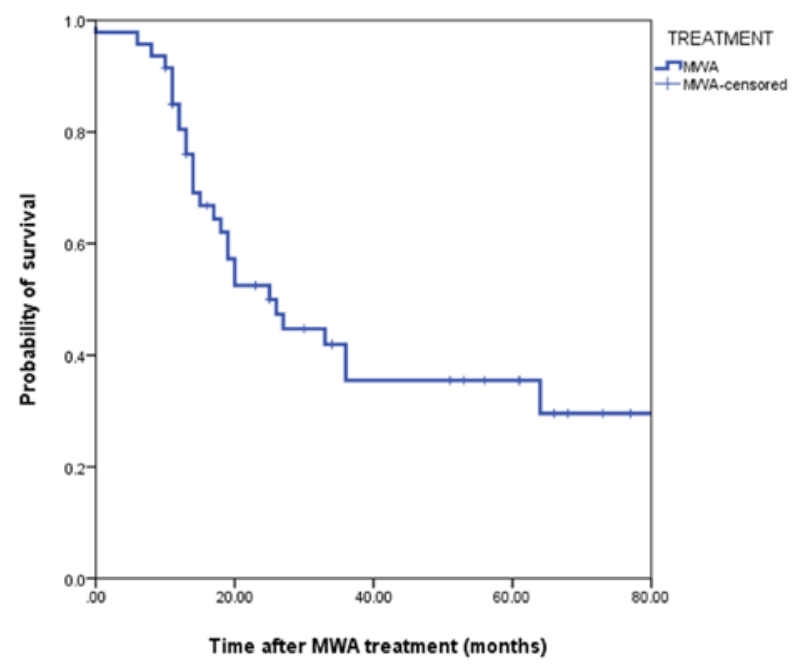

Figure 5. CSS after MWA for non-small-cell lung cancer. MWA, microwave ablation. CSS, cancer-specific survival.

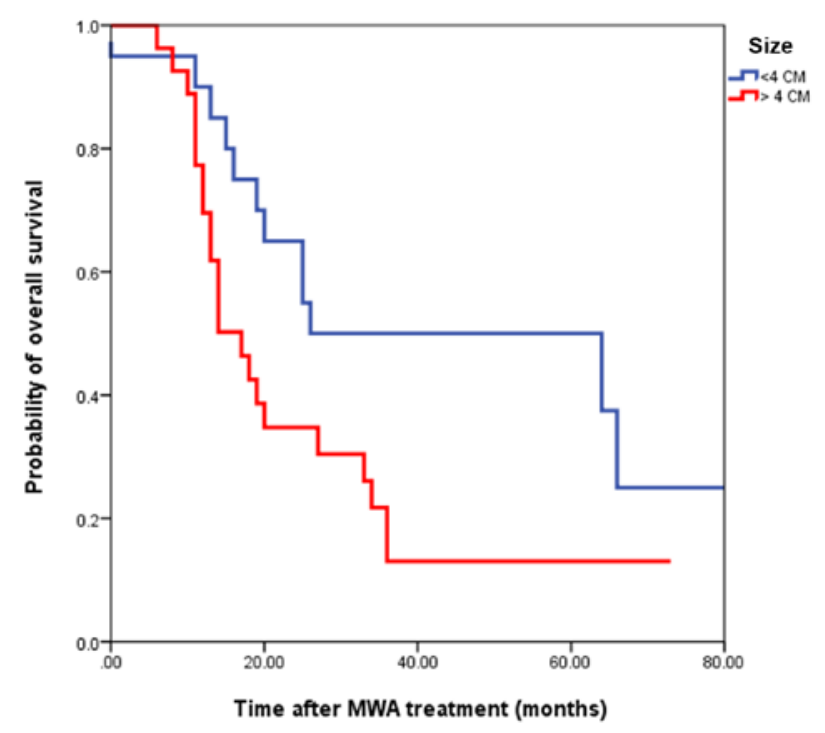

Figure 6. Overall survival after MWA for non-small-cell lung cancer in relation to tumor size. $\mathrm{P}=0.03$ (log rank test). MWA, microwave ablation. 
Table III. Multivariate logistic regression for factors associated with LTP in patients who received microwave ablation for NSCLC.

\begin{tabular}{|c|c|c|c|c|c|}
\hline Variables & Odds ratio & Standard error & $\mathrm{Z}$ score & P-value & $95 \% \mathrm{CI}$ \\
\hline \multicolumn{6}{|c|}{ Complete tumor ablation after the 1 st MWA session } \\
\hline No & 6.57 & 0.95 & 1.88 & $0.048^{\mathrm{a}}$ & $1.02-42.34$ \\
\hline Yes & Ref & & & & \\
\hline \multicolumn{6}{|c|}{ Proximity to relevant structures } \\
\hline Yes & 2.09 & 0.73 & 0.73 & 0.313 & $0.50-8.74$ \\
\hline No & Ref & & & & \\
\hline \multicolumn{6}{|c|}{ Tumor size } \\
\hline$\geq 4 \mathrm{~cm}$ & 0.18 & 0.70 & -1.68 & $0.017^{\mathrm{a}}$ & $0.04-0.74$ \\
\hline$<4 \mathrm{~cm}$ & Ref & & & & \\
\hline
\end{tabular}

${ }^{\mathrm{a}} \mathrm{P}<0.05$. LTP, local tumor progression; NSCLC, non-small cell lung cancer.

treatment $(8,9,24,25)$. These patients are usually treated in a multidisciplinary fashion, with systemic therapies and radiation therapy being the most commonly used modalities. However, all these treatments rarely provide a cure or good long term survival outcomes (8). In this scenario, MWA has been shown to be effective in reducing tumor size in patients with inoperable lung cancer. However, many studies in the literature include patients with both primary and metastatic tumors, thus resulting in limited validity in evaluating survival outcomes in those affected by primary lung cancer $(9,11,15,18,22)$. Moreover, the few studies focusing on MWA of NSCLC mostly include patients with inoperable lesions at an early stage $(4,15,23,26)$. The present study addressed the role of MWA in patients with large advanced NSCLCs, mostly at stage III, who were unsuitable for surgery. In fact, only three patients in this series received MWA because they refused to undergo surgery.

In the present study, MWA was used in patients with NSCLC lesions measuring between 3 and $14 \mathrm{~cm}$. Zhong et al (8), who published one of the largest series of NSCLC patients treated with MWA, included tumor lesions up to $6 \mathrm{~cm}$ in maximum diameter. Likewise, other authors have included only tumors smaller than $4-5 \mathrm{~cm}$ for MWA $(4,9,18)$. Notably, more than one-half of the lesions in the present study were larger than $4 \mathrm{~cm}$, with a mean tumor size of $5 \mathrm{~cm}$. According to the recent literature $(4,18,27)$, no NSCLC lesions $>6 \mathrm{~cm}$ have been treated with MWA. This may be due to the notion that ablation of very large lesions may not be able to obtain complete tumor necrosis. However, the authors of the present study hypothesize that cytoreduction may be of benefit in such situations. Moreover, one of the advantages of MWA is the possibility of treating large tumors by using two or more antennae simultaneously. In these situations partial tumor necrosis is usually achieved after the first session of treatment, and in selected cases a redo-MWA can be carried out to obtain better control of the disease. In the present series, $18.5 \%$ of patients received a second MWA treatment.

A common problem in the application of percutaneous ablation techniques is the proximity of the lesions to relevant anatomical structures, because of the possible heat damage to the surrounding tissues and organs $(15,18)$. Approximately
$17 \%$ of patients included in the present study had NSCLCs in the vicinity of structures such as the aorta, mediastinal pleura, main stem bronchus, pericardium or diaphragm. Notably, in those patients, MWA treatment was completed without any specific consequences. Other recent papers have highlighted a progressive broadening of indications for MWA treatment of lung malignancies located near these structures $(19,28)$.

Although side effects and serious complications related to percutaneous thermoablation can occur (20), in the present study MWA-related complications were observed in $27.7 \%$ of cases, none of which were considered life-threatening for the patients. This data reinforce the concept that MWA of lung tumors is a safe procedure when performed by trained experts $(4,20)$.

Local progression was observed in $35.8 \%$ of patients in the present study. This figure is high compared with other reports. For example, Zhong et al (8), reported a local progression or relapse in $20.5 \%$ of 78 patients undergoing MWA for advanced NSCLC. In general, rates of tumor progression after pulmonary MWA range between 0 and $34 \%$ in the literature (10). The reason for this finding may be due to the large tumor sizes in the present study. In fact the larger the tumor mass, the lower the possibility of obtaining complete necrosis after MWA. It was observed that incomplete tumor ablation after the first MWA session was a significant independent predictor of LTP, according to the multivariate analysis $(\mathrm{P}<0.05)$. Nonetheless, thermal ablation can be repeated after tumor progression (27) and can also be considered as a salvage therapy in cases of local relapse after a previous treatment (15).

Studies on IIIa/IIIb NSCLC cases not receiving MWA showed a 5 -year OS range between 5 and $25 \%$, and a CSS range between 10 and 36\% (10,29-32). In the present report, the 5-year OS was $18.3 \%$, while the 5-year CCS was $30.0 \%$. These data seem to compare favorably with previous published data on survival in patients with locally advanced NSCLC, especially if one takes into account that this study focused on patients with large lesions. To date, no trials have been conducted to compare MWA and non-ablative techniques, and few studies have reported on long term outcomes. As expected, OS in patients with NSCLC $>4 \mathrm{~cm}$ at baseline CT scan was 
significantly lower when compared to those with smaller-size tumors $(\mathrm{P}=0.03)$. The reasons for this finding may involve both the more advanced stage of the disease and the lower probability of obtaining complete tumor necrosis with MWA in the group with larger tumors. Yang et al (23), similarly reported that NSCLC $\leq 3.5 \mathrm{~cm}$ was associated with better survival than tumors $>3.5 \mathrm{~cm}$.

In 12 cases of the present series in which tumor necrosis was incomplete after the first MWA session, the treatment was repeated in order to obtain complete necrosis, and in 3 cases a third session was needed. This confirms that MWA is a versatile ablative method that can be safely repeated in the same patient in cases of incomplete ablation or tumor progression (27).

It was observed that, along with incomplete tumor ablation, a tumor size $\geq 4 \mathrm{~cm}$ was a significant independent predictor of LTP $(\mathrm{P}<0.05)$. Similarly, Zheng et al $(11)$ reported a significant difference in LTP in patients with lung tumors with a mean diameter of $3.1 \mathrm{~cm}$ compared with those with a mean diameter of $4.9 \mathrm{~cm}$. Also, in the study of Lu et al (33) on a group of 69 patients, a higher local progression rate was observed when tumors were $>4 \mathrm{~cm}$ at the baseline CT scan.

The present study had some limitations; firstly, its non-comparative design and the small simple size. Nonetheless, the preliminary results support the utility of MWA as a form of palliative tumor ablation in patients with advanced NSCLC. In particular, to the best of our knowledge this is the first study to report long term follow-up data of MWA in patients with large NSCLC. Although current guidelines comprise the use of interventional radiological ablation as an option for selected patients with stage I NSCLC who are medically inoperable (34-36), the role of MWA thermoablation remains ill-defined. It is considered that further multicenter studies are needed to better assess the role of MWA in local tumor control and to evaluate survival outcomes, as well as to improve its use in integrated multimodality treatment. In addition, promising interactions between MWA and targeted agents have been reported, and this merits further investigation $(15,37,38)$.

In summary, CT-guided MWA may represent an useful tool in the multimodality treatment of patients with large advanced NSCLC. MWA was successfully applied in large NSCLCs in close proximity to relevant anatomical structures.

\section{Acknowledgements}

Not applicable.

\section{Funding}

No funding was received.

\section{Availability of data and materials}

The datasets used and/or analyzed during the present study are available from the corresponding author on reasonable request.

\section{Authors' contributions}

CP and AF contributed to the study conception and design, wrote the manuscript, and analyzed the data. LM and BS were involved in data collection and processing. DG acquired, analyzed and interpreted the oncological data, and drafted the manuscript. AP critically revised the manuscript for important intellectual content and interpreted the oncological data. All authors read and approved the final version of the manuscript.

\section{Ethics approval and consent to participate}

The Institutional Review Board of the Division of Interventional Radiology, Department of Oncological Radiology, Oncological Hospital A. Businco, Cagliari (Italy) approved this study. All patients gave informed written consent for the MWA treatment.

\section{Patient consent for publication}

All patients or their immediate relatives provided consent for publication.

\section{Competing interests}

The authors declare that they have no competing interests.

\section{References}

1. Malvezzi M, Carioli G, Bertuccio P, Boffetta P, Levi F, La Vecchia $C$ and Negri E: European cancer mortality predictions for the year 2017, with focus on lung cancer. Ann Oncol 28: 1117-1123, 2017.

2. Lewis J, Gillaspie EA, Osmundson EC and Horn L: Neoadjuvant approaches to locally advanced non-small cell lung cancer. Front Oncol 8: 5, 2018

3. Ou W, Li N, Wang SY, Li J, Liu QW, Huang QA and Wang BX: Phase 2 trial of neoadjuvant bevacizumab plus pemetrexed and carboplatin in patients with unresectable stage III lung adenocarcinoma (GASTO 1001). Cancer 122: 740-747, 2016.

4. Liu H and Steinke K: High-powered percutaneous microwave ablation of stage I medically inoperable non-small cell lung cancer: A preliminary study. J Med Imaging Radiat Oncol 57: 466-474, 2013.

5. Moya-Horno I, Viteri S, Karachaliou N and Rosell R: Combination of immunotherapy with targeted therapies in advanced non-small cell lung cancer (NSCLC). Ther Adv Med Oncol 10: $1758834017745012,2018$.

6. AmericanCancer Society: Cancer Facts \& Figures. 2017; https://www.cancer.org/cancer/non-small-cell-lung-cancer/detectiondiagnosis staging/survival-rates.html.

7. Nour-Eldin NA, Exner S, Al-Subhi M, Naguib NNN, Kaltenbach B, Roman A and Vogl TJ: Ablation therapy of non-colorectal cancer lung metastases: Retrospective analysis of tumour response post-laser-induced interstitial thermotherapy (LITT), radiofrequency ablation (RFA) and microwave ablation (MWA). Int J Hyperthermia 33: 820-229, 2017.

8. Zhong L, Sun S, Shi J, Cao F, Han X, Bao X and You Q: Clinical analysis on 113 patients with lung cancer treated by percutaneous CT-guided microwave ablation. J Thorac Dis 9: 590-597, 2017.

9. Wolf FJ, Grand DJ, Machan JT, Dipetrillo TA, Mayo-Smith WW and Dupuy DE: Microwave ablation of lung malignancies: Effectiveness, CT findings, and safety in 50 patients. Radiology 247: 871-879, 2008.

10. Vogl TJ, Naguib NN, Gruber-Rouh T, Koitka K, Lehnert T and Nour-Eldin NE: Microwave ablation therapy: Clinical utility in treatment of pulmonary metastases. Radiology 261: 643-651,2011.

11. Zheng A, Ye X, Yang X, Huang G and Gai Y: Local efficacy and survival after microwave ablation of lung tumors: A retrospective study in 183 patients. J Vasc Interv Radiol 27: 1806-1814, 2016.

12. Pusceddu C, Melis L, Ballicu N, Meloni P, Sanna V, Porcu A and Fancellu A: Cryoablation of primary breast cancer in patients with metastatic disease: considerations arising from a single-centre data analysis. Biomed Res Int 2017: 3839012, 2017.

13. Pusceddu C, Melis L, Sotgia B, Fancellu A and Meloni GB: Computed tomography-guided cryoablation of local recurrence after primary resection of pancreatic adenocarcinoma. Clin Pract 5: 741, 2015. 
14. Pusceddu C, Sotgia B, Fele RM and Melis L: Treatment of bone metastases with microwave thermal ablation. J Vasc Interv Radiol 24: 229-233, 2013.

15. Palussière $\mathrm{J}$, Catena $\mathrm{V}$ and Buy $\mathrm{X}$ : Percutaneous thermal ablation of lung tumors-Radiofrequency, microwave and cryotherapy: Where are we going? Diagn Interv Imaging 98: 619-625, 2017.

16. Wang H, Littrup PJ, Duan Y, Zhang Y, Feng H and Nie Z: Thoracic masses treated with percutaneous cryotherapy: Initial experience with more than 200 procedures. Radiology 235: 289-298, 2005.

17. Hegenscheid K, Behrendt N, Rosenberg C, Kuehn JP, Ewert R, Hosten N and Puls R: Assessing early vascular changes and treatment response after laser-induced ther- motherapy of pulmonary metastases with perfusion CT: Initial experience. AJR Am J Roentgenol 194: 1116-1123, 2010.

18. Belfiore G, Ronza F, Belfiore MP, Serao N, di Ronza G, Grassi R and Rotondo A: Patients' survival in lung malignancies treated by microwave ablation: Our experience on 56 patients. Eur J Radiol 82: 177-181, 2013.

19. Pusceddu C, Melis L, Fancellu A, Melis M and Meloni GB: Feasibility and safety of percutaneous radiofrequency, microwave or cryoablation for unresectable thoracic malignancies in close proximity to heart and large vessels. Ann Surg Oncol 20 S107, 2013

20. Zheng A, Wang X, Yang X, Wang W, Huang G, Gai Y and Ye X: Major complications after lung microwave ablation: A single-center experience on 204 sessions. Ann Thorac Surg 98: 243-248, 2014

21. Pusceddu C, Melis L, Ballicu N, Sotgia B, Melis M, Sanna V, Meloni GB, Porcu A and Fancellu A: Percutaneous microwave ablation under CT guidance for hepatocellular carcinoma: A single institutional experience. J Gastrointest Cancer 49: 295-301, 2018.

22. Carrafiello G, Mangini M, Fontana F, Ierardi AM, De Marchi G, Rotolo N, Chini C, Cuffari S and Fugazzola C: Microwave ablation of lung tumors: Single-centre preliminary experience. Radiol Med 119: 75-82, 2014

23. Yang X, Ye X, Zheng A, Huang G, Ni X, Wang J, Han X, Li W and Wei Z: Percutaneous microwave ablation of stage I medically inoperable non-small cell lung cancer: Clinical evaluation of 47 cases. J Surg Oncol 110: 758-763, 2014.

24. Macchi M, Belfiore MP, Floridi C, Serra N, Belfiore G, Carmignani L, Grasso RF, Mazza E, Pusceddu C, Brunese L and Carrafiello G: Radiofrequency versusmicrowave ablation for treatment of the lung tumors: LUMIRA (lung microwave radiofrequency) randomized trial. Med Oncol 34: 96, 2014

25. Lackey A and Donington JS: Surgical management of lung cancer. Semin Intervent Radiol 30: 133-140, 2013.

26. Narsule CK, Sridhar P, Nair D, Gupta A, Oommen RG, Ebright MI, Litle VR and Fernando HC: Percutaneous thermal ablation for stage IA non-small cell lung cancer: Long-term follow-up. J Thorac Dis 9: 4039-4045, 2017.

27. Yang X, Ye X, Huang G, Han X, Wang J, Li W, Wei Z and Meng M: Repeated percutaneous microwave ablation for local recurrence of inoperable Stage I nonsmall cell lung cancer. J Cancer Res Ther 13: 683-688, 2017.
28. Maxwell AWP, Healey TT and Dupuy DE: Microwave ablation of lung tumors near the heart: A retrospective review of short-term procedural safety in ten patients. Cardiovasc Intervent Radiol 40: 1401-1407, 2017.

29. De Tollenaere C, Lievens Y, Vandecasteele K, Vermaelen $K$ and Surmont V: Unresectable stage III non-small-cell lung cancer: Have we made any progress? World J Respirol 5: 140-151, 2015.

30. Stinchcombe TE and Bogart JA: Novel approaches of chemoradiotherapy in unresectable stage IIIA and stage IIIB non-small cell lung cancer. Oncologist 17: 682-693, 2012.

31. Caglar HB, Baldini EH, Othus M, Rabin MS, Bueno R, Sugarbaker DJ, Mentzer SJ, Jänne PA, Johnson BE and Allen AM: Outcomes of patients with stage III nonsmall cell lung cancer treated with chemotherapy and radiation with and without surgery. Cancer 115: 4156-4166, 2009.

32. Wang H, Zhang J, Shi F, Zhang C, Jiao Q and Zhu H: Better cancer specific survival in young small cell lung cancer patients especially with AJCC stage III. Oncotarget 8: 34923-34934, 2017.

33. Lu Q, Cao W, Huang L, Wan Y, Liu T, Cheng Q, Han Y and Li X CT-guided percutaneous microwave ablation of pulmonary malignancies: Results in 69 cases. World J Surg Oncol 10: 80, 2012.

34. NCCN guidelines for Non-Small Cell Lung Cancer (Ver. 4.2018-April 2018). https://www.nccn.org/store/login/login. aspxReturnURL=https://www.nccn.org/professionals/physician_ gls/pdf/nscl.pdf

35. Postmus PE, Kerr KM, Oudkerk M, Senan S, Waller DA, Vansteenkiste J, Escriu C and Peters S; ESMO Guidelines Committee: Early and locally advanced non-small- cell lung cancer (NSCLC): ESMO Clinical Practice Guidelines for diagnosis, treatment and follow-up. Ann Oncol 28 (suppl 4): iv1-iv21, 2017.

36. Howington JA, Blum MG, Chang AC, Balekian AA and Murthy SC: Treatment of stage I and II non-small cell lung cancer: Diagnosis and management of lung cancer, 3rd ed: American College of Chest Physicians evidence-based clinical practice guidelines. Chest 143 (5 Suppl): e278S-e313S, 2013.

37. Wei Z, Ye X, Yang X, Zheng A, Huang G, Li W, Wang J, Han X, Meng M and Ni Y: Microwave ablation combined with EGFR-TKIs versus only EGFR-TKIs in advanced NSCLC patients with EGFR-sensitive mutations. Oncotarget 8: 56714-56725, 2017.

38. Ni Y, Bi J, Ye X, Fan W, Yu G, Yang X, Huang G, Li W, Wang J, Han X, et al: Local microwave ablation with continued EGFR tyrosine kinase inhibitor as a treatment strategy in advanced non-small cell lung cancers that developed extra-central nervous system oligoprogressive disease during EGFR tyrosine kinase inhibitor treatment: A pilot study. Medicine (Baltimore) 95: e3998, 2016.

This work is licensed under a Creative Commons Attribution-NonCommercial-NoDerivatives 4.0 International (CC BY-NC-ND 4.0) License. 